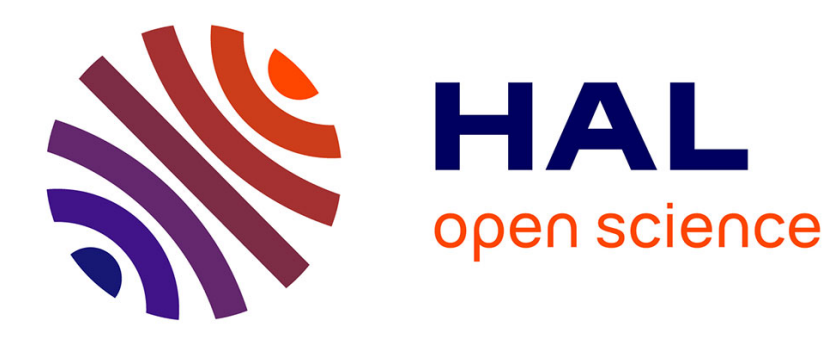

\title{
Le droit à la déconnexion : un premier pas ! ! ! ! Sophie Fantoni-Quinton
}

\section{To cite this version:}

Sophie Fantoni-Quinton. Le droit à la déconnexion : un premier pas ! ! ! !. Archives des Maladies Professionnelles et de L'Environnement, 2017, 78 (6), pp.516 - 518. 10.1016/j.admp.2017.08.007 . hal-01721482

\section{HAL Id: hal-01721482 \\ https://hal.science/hal-01721482}

Submitted on 12 Mar 2018

HAL is a multi-disciplinary open access archive for the deposit and dissemination of scientific research documents, whether they are published or not. The documents may come from teaching and research institutions in France or abroad, or from public or private research centers.
L'archive ouverte pluridisciplinaire HAL, est destinée au dépôt et à la diffusion de documents scientifiques de niveau recherche, publiés ou non, émanant des établissements d'enseignement et de recherche français ou étrangers, des laboratoires publics ou privés. 
Le droit à la déconnexion : un premier pas !!!!

The right to disconnect: a first step !!!!

S Fantoni Quinton, Université Lille2

Lé développement du numérique a bousculé la notion de temps et du lieu de travail. II permet la réactivité, la disponibilité, la flexibilité mais rend la mesure du temps de travail impropre à évaluer son rythme, sa densité, son intensité, donc la charge de travail.

Dans un univers hyper connecté, la frontière entre temps professionnel et temps extraprofessionnel s'estompe, la charge de travail devient plus difficile à évaluer et les risques se multiplient tant pour la santé des salariés eu égard à leur qualité de vie au travail (et hors travail) et à leurs conditions de travail que pour les entreprises.

Pour les salariés, le développement des technologies d'information et de communication, s'il est mal maîtrisé ou mal régulé, peut avoir un impact sur la santé des salariés. Il peut notamment amplifier les facteurs à l'origine de risques psychosociaux (stress, épuisement professionnel, etc.). Parmi eux, la charge de travail et la surcharge informationnelle, le brouillage des frontières entre vie privée et vie professionnelle sont des risques associés à l'usage du numérique.

D'après les résultats d'un sondage TNS Sofres présenté par l'Agence nationale pour l'amélioration des conditions de travail (ANACT) lors de la 11ème semaine pour la qualité de vie au travail du 16 au 20 juin 2014, pour un quart des salariés, leur vie professionnelle empiète fréquemment sur leur vie privée (à noter que parmi les cadres interrogés, cette proportion s'élève à 38\%). Qui plus est, $34 \%$ des salariés estiment que l'équilibre entre leur vie professionnelle et leur vie privée s'est dégradé au cours des dernières années (cette dégradation est encore plus répandue chez les cadres : 39\%).

Pour les entreprises, la sur-sollicitation numérique et la connexion permanente des salariés peuvent certes permettre le contrôle et être motivées par la recherche d'une plus grande performance mais en réalité, ces contraintes peuvent s'avérer néfastes pour l'entreprise : déconcentration des salariés pendant les réunions, interruption permanente du travail par 
des notifications d'e-mails, accumulation de retard, perte d'efficacité... Sans compter le risque contentieux, réel, lié au non-paiement des heures supplémentaires, au non-respect des heures de repos, à la réparation des préjudices du fait de la dégradation des conditions et des relations de travail etc...

II apparaissait donc nécessaire d'encadrer l'utilisation d'outils numériques professionnels, par un droit à la déconnexion. Ce droit est défini pour le salarié comme celui de ne plus être connecté à ses outils numériques (ordinateur, tablette, Smartphone, réseaux filaires etc) en dehors de son temps de travail. Il a d'abord été introduit dans le droit par la jurisprudence et mentionné par certains accords d'entreprise depuis quelques années (1). II a ensuite été consolidé par la Loi travail 2016 dans le cadre de son chapitre relatif à l'adaptation du droit du travail à l'ère du numérique (2) qui laisse finalement une grande marge de manœuvre aux entreprises dans les actions qui peuvent être mises en œuvre et dont nous donnerons des illustrations (3).

\section{Un droit reconnu par la jurisprudence et faisant l'objet d'accords d'entreprise}

Ce principe du droit à la déconnexion avait été pris en compte de manière parcellaire par les partenaires sociaux dans le cadre d'accords interprofessionnels, notamment celui du 19 juin 2013 sur une politique d'amélioration de la qualité de vie au travail et de l'égalité professionnelle qui, en son article 17 " promouvoir une gestion intelligente des technologies de l'information et de la communication au service de la compétitivité des entreprises, respectueuse de la vie privée des salariés » prévoit que les entreprises "rechercheront, après avoir recueilli l'avis des salariés sur I'usage des TIC dans l'entreprise, les moyens de concilier vie personnelle et vie professionnelle en tenant compte des exigences propres aux caractéristiques de l'entreprise et des fonctions exercées, par l'institution, par exemple, de temps de déconnexion, comme cela se pratique déjà dans certaines entreprises ". Pour autant, ce principe n'a jamais fait l'objet en tant que tel de dispositions au niveau interprofessionnel.

Ce sujet a été en fait traité selon des modalités diverses par plusieurs accords d'entreprise.

Le rapport Mettling [1] constate la diversité des accords, par ordre de fermeté croissant : dans certaines entreprises, l'articulation entre vie professionnelle et vie privée est faite tacitement, la porosité étant acceptée et trouvant son équilibre dans l'attachement des 
salariés aux jours de RTT. Dans d'autres entreprises, l'articulation a été formalisée par des accords de type "Droit à la déconnexion ", dont le respect pèse souvent sur le salarié. Certaines d'entre elles ont pris des mesures plus formelles, par exemple par la formalisation expresse du droit du salarié de ne pas répondre aux sollicitations en dehors des plages de travail (ex : La Poste, l'APEC) ou par des expérimentations de modules applicatifs de déconnexion (ex : Orange O’zone). Enfin, certaines entreprises ont décidé de réguler ces pratiques par des dispositifs plus rigides, par exemple en fermant les serveurs de messagerie pendant le week-end. Dans ce cas, c'est l'entreprise qui porte la responsabilité de la régulation.

Pourtant ce rapport précise que $72 \%$ des cadres travaillent dans des entreprises qui n'ont pris aucune mesure de régulation de communication via les outils numériques et plus d'un tiers ont le sentiment de ne bénéficier d'aucun droit à la déconnexion.

Parallèlement, et dans le droit fil de l'obligation de sécurité de résultat de l'employeur qui doit garantir à son salarié des conditions de travail propres à ne pas dégrader son état de santé, le droit à la déconnexion avait été reconnu directement dès 2004 par la Cour de cassation. Ainsi, dans un arrêt du 17 février 2004 la chambre sociale de la Cour de cassation, a estimé que : "le fait de n'avoir pu être joint en dehors de ses horaires de travail sur son téléphone portable personnel est dépourvu de caractère fautif et ne permet donc pas de justifier un licenciement disciplinaire pour faute grave » [2]. Par ailleurs la chambre sociale a souvent condamné des employeurs pour manquement à l'obligation de sécurité de résultat en raison d'une charge de travail excessive en lien ou non avec les outils numériques [3].

Malgré ces évolutions jurisprudentielles, les initiatives qui avaient déjà été menées par certaines entreprises ont été considérées comme trop rares et trop circonscrites au regard de l'enjeu que représente la révolution numérique dans les entreprises et des difficultés courantes liées à l'intrusion des e-mails dans la vie privée. Comme le préconisait le rapport remis par Bruno Mettling, il était donc souhaitable que la Loi aille plus loin. 


\section{Un droit consacré par le Code du travail}

L'option du législateur a été de consacrer ce droit à travers la négociation collective avec les partenaires sociaux, afin que chaque entreprise construise son propre système de gouvernance en fonction de sa culture, de ses métiers, et de ses salariés. Cette question devra obligatoirement être abordée, et ce depuis le $1^{\mathrm{er}}$ janvier 2017, dans le cadre de la négociation annuelle sur l'égalité professionnelle entre les femmes et les hommes et la qualité de vie au travail (Art. L. 2242-8 du Code du travail modifié par la LOI n²016-1088 du 8 août 2016 - art. 55 (V). Cette obligation n'existe donc que dans les entreprises de plus de 50 salariés.

Le $1^{\circ}$ de cet article prévoit que la négociation annuelle porte notamment sur l'articulation entre la vie personnelle et la vie professionnelle pour les salariés ce qui n'est pas dénué de lien avec le droit à la déconnexion qui est pour autant et par ailleurs spécifiquement envisagé dans le point 7. Ce dernier prévoit ainsi que « Les modalités du plein exercice par le salarié de son droit à la déconnexion et la mise en place par l'entreprise de dispositifs de régulation de l'utilisation des outils numériques, en vue d'assurer le respect des temps de repos et de congé ainsi que de la vie personnelle et familiale. A défaut d'accord, l'employeur élabore une charte, après avis du comité d'entreprise ou, à défaut, des délégués du personnel. Cette charte définit ces modalités de l'exercice du droit à la déconnexion et prévoit en outre la mise en œuvre, à destination des salariés et du personnel d'encadrement et de direction, d'actions de formation et de sensibilisation à un usage raisonnable des outils numériques ».

Dans l'étude d'impact de la Loi travail [4], le législateur a estimé que les impacts sociaux de cet article étaient " majeurs » : "Les entreprises devront ainsi réfléchir en fonction de leur organisation à l'impact de ces outils sur son mode de fonctionnement, sur leur mode de management, sur leur mode de production. La prise en compte de la parole des salariés sur ces questions peut permettre de développer le " bien faire son travail 》 et minorer les phénomènes de stress au travail ou d'épuisement professionnel dont les outils modernes ne sont pas toujours la cause mais dont ils peuvent être des amplificateurs importants ». 
La loi est ainsi volontairement non directive dans le contenu même de l'accord, pour laisser à la négociation collective la souplesse nécessaire. Les partenaires sociaux semblent en effet les mieux placés pour discuter de la mise en place des outils de suivi de la charge de travail adaptés à la nature des fonctions occupées par les salariés et de l'activité de l'entreprise ou de la branche.

II faut par ailleurs signaler que le législateur a aussi évoqué en 2016 ce droit à la déconnexion dans le cadre d'un autre article du Code du travail, relatif à la conclusion de conventions individuelles de forfait en jours dans lequel il est notamment prévu que l'accord doit déterminer, outre les modalités selon lesquelles l'employeur assure l'évaluation et le suivi régulier de la charge de travail du salarié, celles selon lesquelles le salarié peut exercer son droit à la déconnexion prévu au $7^{\circ}$ de l'article L.2242-8 (Art. L. 3121-63 II).

3. Les instruments de régulation possibles

Le Code du travail ne fournit pas de notice, de guideline, ou de liste de mesures à mettre en place car le législateur voulait permettre aux entreprises d'innover mais aussi d'être cohérentes avec leurs propres organisations. Cependant, les différents accords, chartes d'entreprises ainsi que les propositions faites par certains spécialistes de l'organisation du travail permettent d'avoir un aperçu des mesures possibles à mettre en place. Les principales initiatives retrouvées sont par exemple :

- Les actions de sensibilisation et de formation (y compris des managers) aux usages numériques pour maitriser les fonctionnalités pour se déconnecter par exemple ou éviter " répondre à tous ",

- Des plages de déconnexion le week-end, le soir et la nuit

- Le droit de ne pas répondre aux e-mails tardifs, le paramétrage des outils numériques en mode « ne pas déranger » lors de certaines heures

- Les fenêtres d'alerte sur les messageries professionnelles proposant lors de l'envoi un envoi différé sauf en cas d'urgence

- Le blocage des serveurs informatiques pendant certaines heures 
- Les études de flux sur la base du volontariat, pour analyser les sur-connexions et proposer des mesures correctives basées sur l'analyse de l'organisation de travail

II semble assez évident que la mise en place de mesures isolées sans information, formation ou sans regard sur l'organisation de travail serait vouée à l'échec car relèverait davantage de l'incantation que d'une volonté réelle de mutation...

En conclusion, le droit à la déconnexion a connu une impulsion certaine avec la Loi travail 2016 et le sillon tracé doit continuer à être labouré pour ne pas se transformer en ornière car l'objectif est de mieux assurer le respect des temps de travail, des temps de repos y compris de congés ainsi que l'équilibre vie professionnelle-vie privée, le droit à la à la qualité de vie au travail et à la santé des salariés. Il s'agit d'un droit et non d'un devoir et l'absence de sanction prévue pourrait dissuader les entreprises d'engager des négociations sur ce terrain ou de rédiger une charte. Malgré l'absence de mesures de coercition, les obligations en matière de sécurité de résultat devraient néanmoins fortement inciter les entreprises à s'emparer de cette question pour s'attaquer au problème en amont, celui de l'organisation collective de travail et de la charge de travail.

\section{Pas de conflit d'intérêt.}

\section{Références bibliographiques}

1. Rapport rédigé par Mr Mettling "Transformation numérique et vie au travail ", sept. 2015, La documentation française, 69p.

2. Arrêt de la chambre sociale de la cour de cassation du 17 février 2004, Nº pourvoi: 0145889.

3. Arrêt de la chambre sociale de la cour de cassation du 21 janvier 2015 , № de pourvoi: $13-$ 16896.

4. Etude d'impact sur la Loi Travail, en ligne sur le site de l'Assemblée nationale : http://travail-emploi.gouv.fr/IMG/pdf/pl3600-ei.pdf consulté le 2 août 2017. 